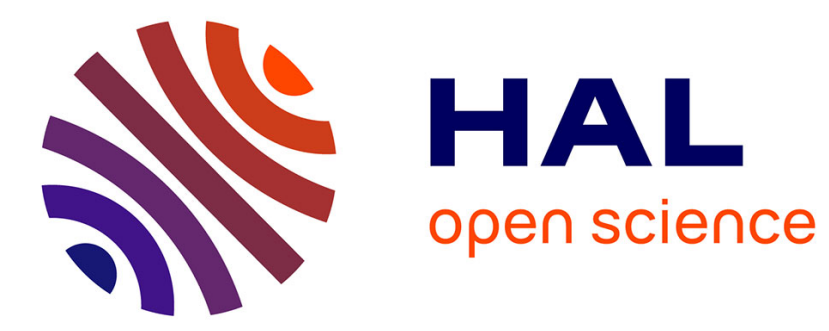

\title{
The UV price for symmetry non-restoration
}

Jean Orloff

\section{To cite this version:}

Jean Orloff. The UV price for symmetry non-restoration. Physics Letters B, 1997, 403, pp.309 - 315. in2p3-00022919

\section{HAL Id: in2p3-00022919 https://hal.in2p3.fr/in2p3-00022919}

Submitted on 17 Dec 2014

HAL is a multi-disciplinary open access archive for the deposit and dissemination of scientific research documents, whether they are published or not. The documents may come from teaching and research institutions in France or abroad, or from public or private research centers.
L'archive ouverte pluridisciplinaire HAL, est destinée au dépôt et à la diffusion de documents scientifiques de niveau recherche, publiés ou non, émanant des établissements d'enseignement et de recherche français ou étrangers, des laboratoires publics ou privés.

\section{다)(1) $(5$}

Distributed under a Creative Commons Attribution - NonCommercial| 4.0 International 


\title{
The UV price for symmetry non-restoration
}

\author{
Jean Orloff ${ }^{1}$ \\ Laboratoire de Physique Théorique, ENSLAPP, Lyon, France ${ }^{2}$ \\ Received 19 December 1996; revised manuscript received 17 April 1997 \\ Editor: L. Alvarez-Gaumé
}

\begin{abstract}
We study the non restoration of symmetries with a local order parameter in field theory at finite temperature. After giving an interpretation of the phenomenon, we show that hierarchy problems are a necessary condition for its realization in renormalizable theories. We then use a large $N$ treatment, and find that high temperature symmetry can stay broken in this limit (in opposition with a previous result), and further that the running of couplings reinforces the effect in the simplest model with two scalars. (C) 1997 Published by Elsevier Science B.V.
\end{abstract}

\section{Introduction}

Since soft pion theorems in the sixties and renormalization of the standard model in the seventies, spontaneous symmetry breaking has become an inevitable building block in particle physics models to understand the small mass of certain scalar particles, or the large mass of certain gauge ones. It was later noted $[1,2]$ that in analogy with ferro-magnetism, heating up such models tends to restore the broken symmetry. There are two related intuitive arguments why this should be expected. The first one appeals to thermodynamics: for higher temperatures, the minimum of the free energy becomes determined less by energy (which has broken symmetry minima), than by entropy (which intuitively is maximum in symmetric configurations). Alternatively, one can say that thermal fluctuations are able to cross the potential barriers surrounding the broken minima, and feel the global

\footnotetext{
${ }^{1}$ E-mail: orloff@lapp.in2p3.fr.

${ }^{2}$ URA 14-36 du CNRS, associée à l'Ecole Normale Supérieure de Lyon et à l'Université de Savoie.
}

symmetry of the theory.

This implies that any symmetry broken today was once unbroken when the cosmological temperature was high enough, and there must have been a phase transition where this freezing of symmetry occurs. During this freezing, various topological defects can be created. Certain of these, like monopoles or domain walls are experimentally excluded, which gives constraints on the particle physics models. For instance, this makes it hard to explain the breaking of discretc symmetries (like CP) dynamically: at the phase transition where the symmetry gets spontaneously broken, domain walls separating the regions with different discrete minima would be formed, and these could quickly dominate the energy density of the universe.

To relax these constraints, it has been noted [3] that domains would not form if the symmetry remains broken, however high the temperature. This is symmetry non-restoration (SNR).

In one of the founding papers of finite temperature field theory, Weinberg [4] already noted that high temperature symmetry restoration was not an unavoidable fate in field theory, and produced the following sim- 
plest example. Consider a scalar theory with 2 fields in the vector representations of an $O\left(N_{1}\right) \otimes O\left(N_{2}\right)$ global symmetry group. The most general renormalizable potential is then:

$$
\begin{aligned}
& V(\phi)=\frac{1}{2} m_{1}{ }^{2} \phi_{1}{ }^{2}+\frac{1}{2} m_{2}{ }^{2} \phi_{2}{ }^{2}+\frac{1}{4} \lambda_{1} \phi_{1}{ }^{4}+\frac{1}{4} \lambda_{2} \phi_{2}{ }^{4} \\
& \quad+\frac{1}{2} \lambda_{12} \phi_{1}{ }^{2} \phi_{2}{ }^{2},
\end{aligned}
$$

for which boundedness at the classical level requires:

$\lambda_{1} \lambda_{2} \geqslant \lambda_{12}^{2} ; \quad \lambda_{1}, \lambda_{2} \geqslant 0$.

Notice this does not exclude a negative mixed coupling $\lambda_{12}$, provided it is not excessively large.

For high enough temperatures and small enough couplings, the effective mass corrections are dominated by the quadratic divergence of the one loop tadpoles:

$$
\begin{aligned}
& m_{2}^{2}(T)=m_{2}^{2} \\
& \quad+\frac{T^{2}}{12}\left[\left(N_{2}+2\right) \lambda_{2}+N_{1} \lambda_{12}\right](1+O(\lambda, m / T))
\end{aligned}
$$

and similarly for $(1 \leftrightarrow 2)$. Thus, for:

$\sqrt{\lambda_{1} \lambda_{2}} \doteq \lambda_{12}^{\max }>-\lambda_{12}>\frac{N_{2}+2}{N_{1}} \lambda_{2}$,

any increase in temperature tends to increase the thermal expectation value of $\phi_{2}$ :

$\left\langle\phi_{2}\right\rangle=\sqrt{\frac{-m_{2}^{2}(T)}{\lambda_{2}}} \sim T$

up to arbitrarily large values, while the potential stays bounded from below.

To get an intuitive understanding of this disturbing result, it helps to plot the equipotentials in the $\left(\phi_{1}, \phi_{2}\right)$ plane. To simplify, we will consider the limit of large fields relevant for high temperatures, and consequently neglect the mass terms in this plot. The thin dashed line is an equipotential for the case of vanishing mixed coupling $\lambda_{12}$. Turning on a negative $\lambda_{12}$, we see the thin plain equipotential grows diagonal extensions that would eventually make the potential bottomless for $-\lambda_{12}>\lambda_{12}^{\max }$. Since all other equipotentials are homothetical to the ones drawn, the minimum of this classical potential lies at the origin. However at finite $T$, we should not only minimize the potential

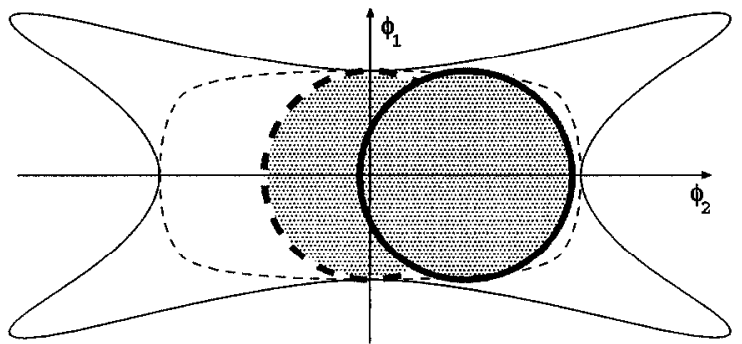

Fig. 1. Contour plots of the classical potentials (1), for $\lambda_{12}=0$ (thin dotted line) and $\lambda_{12}$ close to $-\lambda_{12}$ max (thin plain line). Thick grey disks represent thermal fluctuations about the cold (dotted line) or hot (plain line) vacuum expectation value.

energy for a point, but also for the thermal fluctuations around it, roughly depicted as thick grey disks of size $\sim T$. Because of the pinch in the vertical $\phi_{1}$ direction, the dashed oval get pushed away from the origin, to reach the finite $T$ expectation value surrounded by the plain oval.

With this picture in mind, symmetry non restoration seems to be an indubitable property of certain scalar theories, at least when couplings are small enough that one loop calculations can be trusted. It is thus not surprising that certain crystals (which typically have a rich scalar, vector, tensor, ... excitation spectrum) undergo such an inverse transition where the higher temperature lattice has less symmetry than the lower $T$ one. However, this comparison also calls for a distinction between inverse symmetry breaking and symmetry non restoration. Indeed, by increasing temperature enough, these crystals inevitably melt, thus restoring the full spatial group in the liquid phase. This stresses that any claim for symmetry non restoration is a strong statement about the ultra-violet structure of the theory.

In Section 2, we will see that for inverse symmetry breaking to be possible in a renormalizable theory, its scalar sector must necessarily suffer from hierarchy problems. This excludes renormalizable supersymmetric theories.

In Section 3, we try to see whether there is any sign for symmetry restoration when the model (1) is heated close to the point where its couplings get uncontrollably large (as must somehow happen for crystal excitation modes close to melting). We find contrarywise that the running of couplings seems to increase the symmetry non restoration region. 


\section{The UV price for inverse symmetry breaking: hierarchy problems}

Consider the most general renormalizable field theory, containing scalars $\phi_{i}$, fermions $\psi_{\alpha}$ and vectors $A_{a}$. It is easy to prove that if the theory is hierarchy safe, it cannot undergo inverse transitions. By "hierarchy safe" we here mean the naivest possible definition, namely that relations between dimensionless couplings prevent all dimensional parameters in the lagrangian from receiving power-like divergencies at one loop.

Let us in a first step exclude scalar singlets. To decide whether symmetry is broken or not, we must study the stability of $\left\langle\phi_{i}\right\rangle=0$ which is determined by the lowest non-trivial terms of the potential. Although scalar trilinear couplings exist which could give rise to linear terms at one loop, group theory forces such linear terms to vanish. We must thus focus on mass terms, which by the above hierarchy requirement only receive logarithmic radiative corrections. The quadratic divergencies exactly cancel at one loop between bosons and fermions:

$$
\begin{aligned}
& m_{i}^{2}=\left(c_{i, b}-c_{i, f}\right) \frac{\Lambda_{\mathrm{UV}}^{2}}{16 \pi^{2}}+O\left(\log \Lambda_{\mathrm{UV}}\right) \\
& c_{i, b} \equiv c_{i, f},
\end{aligned}
$$

where $c_{i, b(f)}$ is a combination of all couplings of scalar $i$ to bosons (fermions). Such a cancellation is for instance automatic in supersymmetric theories [5], but the argument here is simpler and more general.

Turning on temperature, we find:

$$
\begin{aligned}
& m_{i}^{2}(T)-m_{i}^{2}(0)=c_{i, t} \frac{T^{2}}{12}+c_{i, f} \frac{T^{2}}{24}+O(\log T) \\
& =3 c_{i, f} \frac{T^{2}}{24}+O(\log T),
\end{aligned}
$$

using "hierarchy safety" to obtain the last line. Contrary to bosonic contributions like (3), $c_{i, f}$ can easily be checked to be positive semi-definite. Indeed, they are the cigenvalucs of a mass matrix $g_{i j}$ of the form:

$g_{i j}=\sum_{a, b} y_{i a h} y_{j a b}^{*} \doteq \sum_{A \doteq(a, b)} y_{i A} y_{j A}^{*}$,

which can be seen as the metric induced on the space of bosonic fields $i$, from the identity metric $\delta_{A B}$ in the space of fermionic pairs $A$, by the linear embedding provided by Yukawa couplings $y_{i A}$. By suitable rotations in the space of bosonic fields, this hermitian matrix can always be diagonalized:

$g_{i j}=\delta_{i j} g_{i}=\delta_{i j} \sum_{A}\left|y_{i A}\right|^{2} \sim \delta_{i j} c_{i, f}$.

This proves that $c_{i, f}$ are positive as claimed, and furthermore, that they can only vanish for scalars totally decoupled from all fermions. Thus, $\left\langle\phi_{i}\right\rangle=0$ is a local minimum, whose stability increases as temperature is raised.

Let us now consider scalar singlets, which allow for new types of linear and trilinear vertices. The only way these can alter the previous conclusion is by having some singlets developping a quadratically divergent expectation value which could contribute to (6) through trilinear coupling. However, this is forbidden by our naive definition of hierarchy safety as it induces an even stronger problem than the usual one for scalar masses. One can for instance show that certain fermions then necessarily receive quadratically divergent contributions to their mass. Hierarchy problems in the scalar sector are thus necessary conditions to have symmetry non-restoration, or even just inverse symmetry breaking.

\section{Gap equations and running couplings}

Let us now focus back on Weinberg's simple model (1) and consider the question of coupling running. Neglecting mass terms, the zero temperature one-loop renormalization of the couplings reads $\left(d t \doteq d \log \Lambda_{U V} / 16 \pi^{2}\right):$

$$
\begin{aligned}
& \frac{d \lambda_{1}}{d t}=\left(N_{1}+8\right) \lambda_{1}(t)^{2}+N_{2} \lambda_{12}(t)^{2} \\
& \frac{d \lambda_{2}}{d t}=\left(N_{2}+8\right) \lambda_{2}(t)^{2}+N_{1} \lambda_{12}(t)^{2}, \\
& \frac{d \lambda_{12}}{d t}=\lambda_{12}(t)\left[\left(N_{1}+2\right) \lambda_{1}(t)+\left(N_{2}+2\right) \lambda_{2}(t)\right. \\
& \left.\quad+4 \lambda_{12}(t)\right] .
\end{aligned}
$$

Independently of the sign of $\lambda_{12}$, all couplings thus tend to grow stronger with increasing $\Lambda_{U V}$, just like for a single self-coupled scalar. As implicd by triviality [6] in the latter case, the theory is only defined below 
$\Lambda_{\text {max }}$, some physical maximum UV cut-off, where the couplings start blowing up.

The presence of such a cut-off requires some care in the definition of symmetry non-restoration. Indeed, the existence of a maximal energy scale means that symmetry (non-) restoration can only be probed up to that temperature, and no easy claim can be done about what happens beyond, where the theory becomes intractable ${ }^{3}$. Furthermore, an upper cut-off opens new possibilities: taking for instance a single scalar with $-m^{2}(T=0) \gg \lambda \Lambda_{\max }^{2}$ will forbid symmetry restoration up to $T \leqslant \Lambda_{\max }$. This trivial effect is however qualitatively different from what we have with (3), where a scalar thermal expectation value constantly increases with temperature.

We will thus focus our attention on the limit $T \gg$ $m(T=0)$, for which we may as well take vanishing masses to start with, and will call symmetry non restoration in the presence of a UV cut-off, the possibility that the symmetry breaking thermal expectation value shows no tendency to decrease as temperature is increased all the way up to the cut-off. In the simplest approach (3), this happens provided a condition on the couplings is satisfied:

$C\left(\lambda_{1}, \lambda_{2}, \lambda_{12}\right)=\left(N_{2}+2\right) \lambda_{2}+N_{1} \lambda_{12}<0$.

However, if couplings are larger, the thermal masses (3) get comparable with $T$. To keep properly track of $O(m / T)$ terms, (3) can be replaced, in the large $\left(N_{1}, N_{2}\right)$ limit, by self-consistent gap equations [7], also known as bubble, super-daisy, or cactus resummation:

$x_{1}^{2} \doteq \frac{m_{1}^{2}(T)}{T^{2}}=\frac{\left(N_{1}+2\right)}{12} \lambda_{1} f\left(x_{1}\right)+\frac{N_{2} \lambda_{12}}{12} f\left(x_{2}\right)$,
$x_{2}^{2} \doteq \frac{m_{2}^{2}(T)}{T^{2}}=\frac{\left(N_{2}+2\right)}{12} \lambda_{2} f\left(x_{2}\right)+\frac{N_{1} \lambda_{12}}{12} f\left(x_{1}\right)$,

where:

$$
\begin{aligned}
& f(x)=\frac{6}{\pi^{2}} \int_{0}^{\infty} \frac{p^{2} d p}{\sqrt{p^{2}+x^{2}}}\left(e^{\sqrt{p^{2}+x^{2}}}-1\right)^{-1} \\
& \sim 1 \quad \frac{3}{\pi} x+O\left(x^{2}\right)
\end{aligned}
$$

\footnotetext{
${ }^{3}$ Notice this equally casts doubts on the restoration of the symmetry above that temperature
}

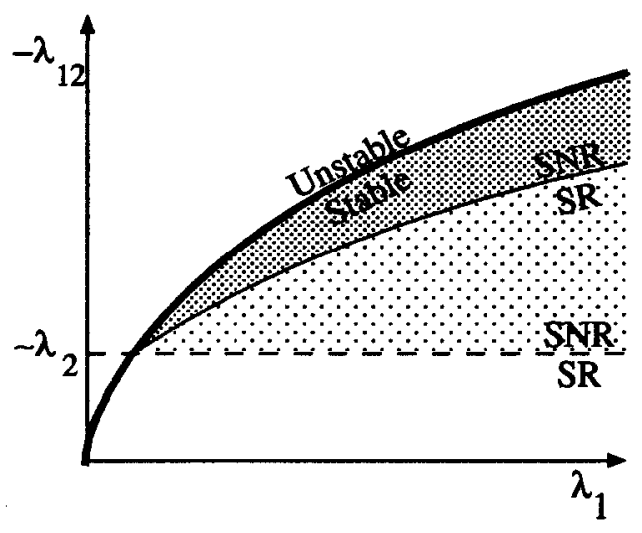

Fig. 2. The Symmetry Non Restoration (SNR) domain in couplings space: the light grey area is given by naive one-loop calculation; the dark grey region is what remains according to gap equations; all stable models should lie below the thick curve.

is exponentially decreasing for large $x$. The approximation $f=1$ just gives back (3), while expanding $f(x)$ around the origin gives perturbative corrections for any $N[4,8,9]$. The new region of symmetry non restoration is now bounded by $x_{2}=0$ which, upon using (12) and dropping $1 / N$ corrections, becomes:

$$
\begin{aligned}
& C\left(\lambda_{1}, \lambda_{2}, \lambda_{12}\right) \\
& \quad=\lambda_{1}-\frac{\lambda_{12}^{2}}{\lambda_{2}}+\frac{12 \lambda_{12}}{N_{2} \lambda_{2}} f^{-1}\left(-N_{2} \lambda_{2} / N_{1} \lambda_{12}\right)^{2} \\
& \quad<0 .
\end{aligned}
$$

This condition on the couplings contrasts with [7], which claims the SNR region to be empty in the same large $N$ limit. The origin of this difference is that we kept all terms in (12) that stay finite in the limit $N_{1,2} \rightarrow \infty$ with $\lambda_{1,2,12} N_{1,2}$ finite. One can further explicitly check that there is no $x_{2}>0$ solution inside the region (14).

Notice that, just like (11), (14) purely gives a condition on the couplings which are kept constant in the gap equations approach. Gap equations can not produce a critical temperature because they have no energy scale other than $T$ in the large temperature limit.

Taking couplings evolution into account will bring in the scale $\Lambda_{\max }$ alluded above. To get a feeling for the direction in which this might go, let us notice that thermal masses come predominantly from "hard" momenta of order $T$ in expressions like (13). It is therefore natural to take the couplings showing up in (12) 
as the running couplings at scale $T$. For a given theory, fixed e.g. by the values of "bare" couplings at a scale as close as reasonable to $\Lambda_{\max }$, the running from that initial scale down to $T$ is close to the running we would have at zero temperature. Indeed, the largest effects of temperature show up in the infra-red, for scales lower than $T$ where the running effectively becomes 3 dimensional [10]. We can therefore tentatively use the same equations (10) and now interpret $d t$ as $d \log T / 16 \pi^{2}$. This procedure was recently legitimated, at least for small couplings [11].

It is interesting to notice that the boundary of the stability region $\lambda_{1} \lambda_{2}=\lambda_{12}^{2}$ is invariant under the large $N$ running of couplings. This is reassuring about the use of the same condition at all scales. Indeed, the global stability of a theory cannot change by integrating out degrees of freedom. Hence, the finite $N$ result that the flows of $\lambda$ can cross the "stability" boundary must mean that operators higher than quartic become relevant to this question [12].

At every temperature, the symmetry non restoration region is thus given in terms of a condition on the running couplings at that temperature. We will now show that increasing $T$ brings new points in this region, so that symmetry non restoration is effectively enhanced by couplings running. This region is bounded by the invariant stability condition and by (14):

$\lambda_{1}=\lambda_{1 c}\left(\lambda_{12}, \lambda_{2}\right) \doteq \frac{\lambda_{12}^{2}}{\lambda_{2}}-\frac{\lambda_{12}}{N_{2} \lambda_{2}} g\left(-N_{2} \lambda_{2} / N_{1} \lambda_{12}\right)$,

where $g(x) \doteq f^{-1}(x)^{2}$. Let us compare the evolution of $\lambda_{1}$ as given by (10), with the evolution along the surface given by:

$$
\begin{aligned}
\frac{d \lambda_{1 c}}{d t} & \doteq \frac{\partial \lambda_{1 c}}{\partial \lambda_{12}} \frac{d \lambda_{12}}{d t}+\frac{\partial \lambda_{1 c}}{\partial \lambda_{2}} \frac{d \lambda_{2}}{d t} \\
& =N_{1} \lambda_{1 c}^{2}+N_{2} \lambda_{12}^{2}+\frac{\lambda_{12}}{N_{2} \lambda_{2}} g^{\prime} g\left(-\frac{N_{2} \lambda_{2}}{N_{1} \lambda_{12}}\right) \\
& =\frac{d \lambda_{1}}{d t}+\frac{\lambda_{12}}{N_{2} \lambda_{2}} g^{\prime} g\left(-\frac{N_{2} \lambda_{2}}{N_{1} \lambda_{12}}\right) .
\end{aligned}
$$

The last term of this expression is positive definite, since $\lambda_{12}$ is negative, and $g$ is purely decreasing,just as $f$ and $f^{-1}$. As a result, when increasing $t$, a point on the surface $\lambda_{1 c}\left(\lambda_{12}, \lambda_{2}\right)$ is moved to a point with a smaller $\lambda_{1}$ than its projection on the surface. This means that the domain of symmetry non restoration increases with temperature.

Let us now critically examine the approximations used to obtain this result, in comparison with the exact renormalization group equations used in [11]. We have first neglected the running of masses as a function of the momentum scale in the gap equations. Taking this into account would change the function $f(x)$ in (13), but it could not change its purely decreasing behavior, which was the only information needed to reach our conclusion.

We have further neglected masses in the running of couplings (10). These would introduce different factors for the loops with different masses. However, the remarkable cancellations leading to (16) would still work, leaving just an $m_{1}$-depending factor in the last term of that equation. Since the bare theory needs a large negative $m_{1}^{2}$ to achieve a small infrared $m_{1}^{2}$, this factor is dominated in the large $N$ limit by the $N_{1}-1$ massless Goldstone modes, for which (16) is thus unaltered.

Finally we have kept couplings fixed in the gap equations. This is a good approximation if couplings evolve slower than masses, like at zero temperature where their logarithmic running is negligible compared with the power-like mass evolution. However this assumption clearly breaks down when nearing the triviality pole. It should be noted that for $T \sim \Lambda_{\max }$, we loose control over the theory anyway, as unavoidable regulator dependencies start creeping in. This shows up in the exact renormalization group approach [11] under the form of strong oscillations in the $T$ dependence when $T / \Lambda_{U V}$ is not small. These prevent any firm conclusion in this region, and the approximation made at least has the merit of cutting them. Going beyond our approximation requires either knowledge of the theory beyond $\Lambda_{\max }$, or at least a way to reach higher temperatures. This is possible by introducing a different spatial and temporal cut-off. The spatial one is still limited by the same $\Lambda_{\max }$, but the temporal cut-off can now be separately sent to infinity, thus allowing for arbitrarily large temperatures. Physically, this corresponds to measuring temperature by the tiny energy dependence in the distribution of modes much smaller than $T$. It has been proven on the lattice [13] that this leads to symmetry restoration. Although this is far from the continuum limit, we expect a similar conclusion with a momentum cut-off. 
Outside of this admittedly artificial regularization procedure, the theory can only be defined beyond $\Lambda_{\text {max }}$ by introducing new physics which may restore the symmetry, or not. The only known fact is that in the latter case, the hierarchy problems get worse beyond $\Lambda_{\max }$.

\section{Conclusion}

In this paper we studied the non restoration of symmetries (SNR) with local order parameters. We showed that in the framework of renormalizable theories, inverse symmetry breaking (and thus a fortiori SNR) necessarily rested on a hierarchy problem for the scalar field associated with the broken order parameter. This excludes renormalizable supersymmetric theories. It has recently been shown [14] that including non-renormalizable operators does not remove this constraint.

We have then shown that in the simplest model of Weinberg (1), the inclusion of running couplings does not lead to symmetry restoration, even when venturing into the ultraviolet strong coupling regime by means of large $N$ techniques. On the contrary, the running of couplings tends to enlarge the region of SNR, in accordance with the recent result of [9] on the effect of next to leading order corrections, the first ones sensitive to coupling running.

This all should not obscure the fact that this model possesses an intrinsic maximum energy scale, which translates into a maximum temperature. Such a maximum scale is quite general in theories with scalars, and is for instance unavoidable when the scalars fully break all non-Abelian gauge symmetries [15]. Without pretending to too much generality, we can say we failed to construct a model with SNR where all couplings enjoy asymptotic freedom. The difficulty lies in that the scalar couplings must be large enough to dominate certain scalar self-energies.

This maximum scale poses a problem for the application of SNR to eliminate topological defects in cosmology. Since classical cosmology should be defined for temperatures up to the Planck scale, we have the following dilemma. Either we tune parameters so that the maximum scale is pushed above the Planck one. In that case, it could be argued that the classical universe is born in the broken phase, but one should somehow cope with graviton loops, and show they do not alter this statement. Or SNR only holds up to some temperature below the Planck scale. In that case, inflation is needed to dispose of the topological defects created at that temperature, and the role of SNR is just to push the moment where these defects appear back in time, carlicr than inflation. However, the higher in encrgy we push them up, the more critical the necessary hierarchy problem becomes. This seems an unavoidable ultra-violet price for SNR.

To conclude, let us stress that we did not touch here symmetry realizations with a non-local order parameter. For these, the picture is totally different. For instance, a duality transformation can exchange high and low temperatures. However, the order parameter broken in the high temperature phase is then nonlocal. Similarly, the Polyakov loop is a non-local order parameter for the deconfining phase transition, and its high temperature thermal expectation value breaks a center $Z_{N}$ symmetry, albeit in a controversial way $[16,17]$.

\section{Acknowledgements}

It is a pleasure to thank B. Gavela, D. Jungnickel, M. Pietroni, N. Rius and C. Wetterich for most informative and enjoyable discussions. It is not a pleasure to thank the bag thief that got me to rewrite and simplify the computations.

\section{References}

[1] D.A. Kirzhnits and A.D. Linde, Macroscopic consequences of the weinberg model, Phys. Lett. B 42 (1972) 471.

[2] D.A. Kirzhnits and A.D. Linde, A relativistic phase transition, Sov, Phys. JETP. 40 (1975) 628.

[3] Gia Dvali and Goran Senjanovic, Is there a domain wall problem?, Phys. Rev. Lett. 74 (1995) 5178.

[4] Steven Weinberg, Gauge and global symmetries at high temperature, Phys. Rev. D 9 (1974) 3357.

[5] Michelangelo Mangano, Global and gauge symmetries in finite temperature supersymmetric theories, Phys. Lett. B 147 (1984) 307.

[6] R. Fernandez, J. Frohlich and A.D. Sokal, Random walks, critical phenomena, and triviality in quantum field theory.

[7] Yasushi Fujimoto and Susumu Sakakibara, On symmetry nonrestoration at high temperature, Phys. Lett. B 151 (1985) 260.

[8] G. Bimonte and G. Lozano, On symmetry nonrestoration at high temperature, Phys. Lett. B 366 (1996) 248. 
[9] Giovanni Amelino-Camelia, Rayleigh-ritz variational approximation and symmetry nonrestoration, Phys. Lett. B 388 (1996) 776.

[10] N. Tetradis and C. Wetterich, The high temperature phase transition for $\phi^{4}$ theories, Nucl. Phys. B 398 (1993) 659.

[11] Thomas G. Roos, Wilson renormalization group study of inverse symmetry breaking, Phys. Rev. D 54 (1996) 2944.

[12] N. Rius M. Pietroni and N. Tetradis, Inverse symmetry breaking and the exact renormalization group, CERNTH/96-341.

[13] G. Bimonte and G. Lozano, Symmetry non restoration and inverse symmetry breaking on the lattice, Phys. Lett. B 388 (1996) 692.
[14] Borut Bajc, Alejandra Melfo and Goran Senjanovic, On supersymmetry at high temperature, Phys. Lett. B 387 (1996) 796.

[15] T.P. Cheng, E. Eichten and Ling-Fong Li, Higgs phenomena in asymptotically free gauge theories, Phys. Rev. D 9 (1974) 2259.

[16] A.V. Smilga, Are $z(n)$ bubbles really there?, Ann. Phys. 234 (1994) 1.

[17] Ian I. Kogan, Gordon W. Semenoff and Nathan Weiss, Induced qcd and hidden local $z(n)$ symmetry, Phys. Rev. Lett. 69 (1992) 3435. 